\title{
Structure and magnetic properties of ultrathin iron films deposited on the CoGa(100) surface
}

\author{
Torsten Balster, Domokos A. Kovacs, Christian Pflitsch, Laurens K. Verheij, * Rudolf David, and René Franchy \\ Institut für Schichten und Grenzflächen (ISG 3), Forschungszentrum Jülich, D-52425 Jülich, Germany
}

(Received 13 May 2002; published 8 November 2002)

\begin{abstract}
Epitaxial bec $\alpha$ - $\mathrm{Fe}(100)$ films are grown on the (100) surface of a CoGa crystal. The misfit between the Fe and the CoGa lattices is very small $(<0.5 \%)$. Both, at room temperature and at $550 \mathrm{~K}$ layer by layer growth is found with thermal energy helium atom scattering (TEAS). The TEAS experiments show that iron films grown at $550 \mathrm{~K}$ on reconstructed $c(4 \times 2) \mathrm{CoGa}(100)$ are smooth. The iron films are stable up to a temperature of 650 $\mathrm{K}$. The magnetic properties of the Fe films are investigated with the magneto-optical Kerr effect. Fe films with a thickness in excess of 1.1-1.3 ML are ferromagnetic at $300 \mathrm{~K}$, with the easy axis for magnetization oriented parallel to the surface. It is found that the coercive field $H_{c}$ is sensitive to the order of the film. Upon annealing at 550-600 K subsequent to deposition, the coercive field, measured around room temperature, increases. The ordering of the film appeared to have no effect on the remanent magnetization. For a 1.7 ML Fe film a Curie temperature of $525 \mathrm{~K}$ is measured. At higher coverage the Curie temperature increases to above $600 \mathrm{~K}$. The coercive field $H_{c}$ is found to approach zero at a 50-100 K lower temperature.
\end{abstract}

DOI: 10.1103/PhysRevB.66.184406

PACS number(s): 75.70.Ak, 68.49.Bc, 68.55.-a

\section{INTRODUCTION}

The growth of ultrathin magnetic films allows the stabilization of new materials, which show different magnetic behavior from that observed for bulk crystals. The main reasons for the deviating behavior are the interaction of the magnetic material with the substrate, the reduced symmetry at the surface, the smaller coordination number in the film, and the lattice strain, which is, in general, induced by the lattice misfit between the film and the nonmagnetic substrate. Iron films are particularly suited for such investigations, since epitaxial films with different lattices (bcc, fcc) and strains have been grown on a variety of substrates. Relatively smooth and well-defined bcc Fe films with little strain have been grown on $\mathrm{Ag}(100)$ (misfit $0.8 \%$ ) and $\mathrm{Au}(100)$ (misfit $0.6 \%$ ). At $300 \mathrm{~K}$, iron grows on the $\operatorname{Ag}(100)$ surface in the layer by layer mode after deposition of around $4 \mathrm{ML} .^{1,2}$ However, STM experiments show that the room-temperature grown film is covered by hillocks with a height of about 1 $\mathrm{nm} .{ }^{3}$ Upon annealing at $600 \mathrm{~K}$ the surface smoothens, the lateral dimension of the islands increases, and the roughness decreases to about $0.4 \mathrm{~nm}(2-3 \mathrm{ML})$. The smoothening is accompanied by $\mathrm{Ag}$ atoms floating on top of the film. Fe films grown at $300 \mathrm{~K}$ on $\mathrm{Au}(100)$ surfaces are smoother than those grown on $\operatorname{Ag}(100)$ as evidenced by reflection highenergy electron diffraction and scanning tunnel microscope (STM) experiments. ${ }^{4,5}$ As the growth temperature increases, double layer islands and pinholes are found until at $400 \mathrm{~K} \mathrm{Au}$ atoms start to float on top of the Fe film. Smooth films are obtained in this way indicating that the gold atoms are acting as surfactant. ${ }^{6}$ Intermixing of the iron film with the substrate is observed above $650 \mathrm{~K}$ for $\mathrm{Ag}(100)$ (Ref. 3) and above 570 $\mathrm{K}$ for $\mathrm{Au}(100){ }^{7} \mathrm{On} \mathrm{Ag}(100)$, in plane remanent magnetization was found at room temperature for Fe films with a thickness in excess of $2.5 \mathrm{ML}$, and on $\mathrm{Au}(100)$ for Fe films with a thickness in excess of 1 ML. ${ }^{8,9}$ In general, no remanent magnetization perpendicular to the surface is observed, though $\mathrm{Fe}$ films with such magnetic behavior have been prepared. ${ }^{10,11}$
Alternative substrates, on which one could expect that smooth and epitaxial bcc Fe films with little strain can be grown, are the alloys $\mathrm{CoGa}$ (misfit $0.5 \%$ ) and $\mathrm{CoAl}$ (misfit $0.2 \%$ ). Both $\mathrm{CoGa}$ and $\mathrm{CoAl}$ are nonmagnetic and have a $\mathrm{CsCl}$ structure, i.e., a bcc-like structure. Here we report on the structural and magnetic properties of an iron film epitaxially grown on $\mathrm{CoGa}(100)$. The clean $\mathrm{CoGa}(100)$ surface shows a $c(4 \times 2)$ reconstruction in two domains, which seems to originate from a $\frac{1}{4}$ ML Co terminating the more or less bulklike CoGa crystal. ${ }^{12,13}$ The growth of 1-10 ML Fe films on this surface was investigated by the scattering of thermal He atoms (TEAS), by low-energy electron diffraction (LEED), and by auger electron spectroscopy (AES). The magnetic properties of the films were studied by the magneto optical Kerr effect (MOKE).

\section{EXPERIMENTAL}

The experiments were performed in two UHV systems, one equipped with TEAS, AES, and LEED, in which the structure and growth phenomena were studied, and a second one equipped with MOKE, AES, and LEED, which was mainly used for the magnetic characterization of the $\mathrm{Fe}$ films. The base pressure in both systems is below $10^{-10}$ mbar. The sample used as a substrate for the Fe films was a $\mathrm{CoGa}(100)$ single crystal in the form of a disk, $10 \mathrm{~mm}$ in diameter and 3-mm thick, which was polished and aligned to within $0.1^{\circ}$. STM experiments performed on similarly prepared surfaces show that the width of the substrate terraces is typically $50-100 \mathrm{~nm} .{ }^{14}$ Cleaning of the $\mathrm{CoGa}(100)$ samples was performed by a combination of $\mathrm{Ar}^{+}$sputtering, oxidation, and annealing at $1100 \mathrm{~K}$, and it was checked by AES. On a freshly polished sample, a well ordered CoGa(100) surface showing a $c(4 \times 2)$ reconstruction is readily prepared. ${ }^{12,13,15}$ After $40-100$ cleaning cycles ( 2-5 months) the sample is found to deteriorate as evidenced by the observation that preparing a $c(4 \times 2)$ reconstructed $\mathrm{CoGa}(100)$ surface becomes more difficult. Whenever this happened, the sample was repolished. Iron was deposited 


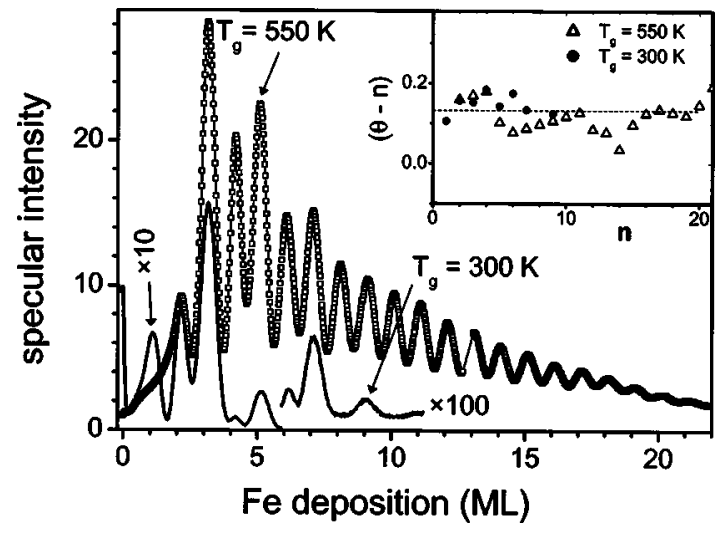

FIG. 1. Deposition of Fe on $\mathrm{CoGa}(100)$ at 300 and $550 \mathrm{~K}$ monitored by specular helium scattering under antiphase conditions. The scattering angle is $90^{\circ}$, the He-beam energy is $31 \mathrm{meV}$. The inset shows the offset of the Fe coverage $\theta$ at the oscillation maxima with respect to a coverage with $n$ layers $(\theta-n)$ as a function of $n$, where $n$ is the number of the oscillation maximum.

from a rod, $2 \mathrm{~mm}$ in diameter, which was heated by electron impact. In all experiments, the deposition rate is around 0.01 $\mathrm{ML} / \mathrm{s}$. Both LEED and TEAS show that an epitaxial bcc $\alpha$-Fe(100) film grows on $\mathrm{CoGa}(100)$. During the deposition of iron, oscillations of the intensity of specularly reflected helium atoms were observed, which allow a very accurate calibration of the deposition rate. The energy of the He atoms in this experiment is $31 \mathrm{meV}$, and the scattering angle is $90^{\circ}$. For this energy and scattering geometry one has destructive interference (antiphase) for the scattering of the atoms from terraces with a height difference of $0.144 \mathrm{~nm}$, corresponding to the Fe step height. In the MOKE system, the deposition rate was calibrated in situ with a quartz microbalance. On the surface of the Fe film we observe a $c(2 \times 2)$ superstructure which is gradually emerging at a deposition between 4 and 7 ML. This structure seems to be due to a small amount of $\mathrm{Ga}$ floating on top of the film. Since the $c(2 \times 2)$ structure is found in both UHV systems to emerge in the same coverage range, we conclude that the two deposition calibrations are equal, within the experimental accuracy. The accuracy of the deposition measurement in the MOKE system is estimated to be about $\pm 15 \%$. The maximum field strength which could be obtained in the MOKE experiments was \pm 600 Oe.

\section{RESULTS}

\section{A. Film growth}

The growth of Fe films on $\mathrm{CoGa}(100)$ was studied with TEAS at temperatures between 300 and 550 K. Figure 1 shows the specular helium intensity during growth at 300 and at $550 \mathrm{~K}$. In the $550 \mathrm{~K}$ growth measurement the intensity is normalized to the specular intensity measured on the clean $\mathrm{CoGa}(100)$ surface (zero Fe deposition). In the $300 \mathrm{~K}$ growth experiment, the intensity is normalized to 10 times, and above $5 \mathrm{ML}$ to 100 times, the intensity at zero Fe coverage. At the growth temperature $T_{g}=300 \mathrm{~K}$ an oscillating intensity of the specular beam under antiphase conditions is observed during $\mathrm{Fe}$ deposition, showing that the film grows in the layer by layer mode. The intensity maxima are equidistant as can be seen in the inset of Fig. 1, where we have plotted $(\theta-n)$ as a function of $n$ whith $\theta$ the Fe deposition in ML and $n$ the number of the oscillation maximum. One may note that the maxima are not found at integer numbers of deposited monolayers but that they are shifted about 0.15 ML to a higher deposition. Except for the peak at $3 \mathrm{ML}$, all peak intensities are lower than the intensity measured at zero coverage. The oscillation at $9 \mathrm{ML}$ can still be resolved in the 300 $\mathrm{K}$ growth curve, but the amplitudes for $n>3$ are very small. An interesting observation is that the odd numbered peaks are much higher than their even numbered neighbors, except for the first one. The fact that we observe the even numbered peaks shows that the growth is really single layer by single layer. The observation of a difference in height of the even and odd numbered peaks shows that the deposited iron atoms are sensing differences between the surfaces of films with an odd or an even number of Fe layers.

Higher intensities are measured and more maxima can be resolved when the deposition temperature is increased. At a deposition temperature $T_{g}=550 \mathrm{~K}$ the highest maximum, observed at a film thickness of $3 \mathrm{ML}$, is about 30 times larger than the specular intensity measured on clean CoGa(100), whereas at $300 \mathrm{~K}$ this maximum is only $50 \%$ larger. The first maximum (1 ML) cannot be resolved in the $550 \mathrm{~K}$ growth curve. Instead of a maximum, as observed at $300 \mathrm{~K}$, we observe a shoulder at a deposition of $1 \mathrm{ML}$. The observation of the shoulder shows that the Fe film is growing, at least to some extent, in a layer by layer mode already at this film thickness. However, at the same time the surface properties are changing drastically as evidenced by the increase of the reflectivity for helium atoms by about a factor of 3 per deposited layer during the growth of the first three layers. This increase of the reflectivity is masking the oscillation at $1 \mathrm{ML}$ to some extent. At a deposition of $2 \mathrm{ML}$ a clear maximum is observed, but the height of this maximum is still low compared to the peaks at higher coverage.

For films with a thickness larger than $2 \mathrm{ML}$, almost normal layer by layer growth behavior is observed at $550 \mathrm{~K}$. Equidistant peaks are found for a film thickness between 3 and 20-25 ML, as can be seen in the inset of Fig. 1. The amplitude of the oscillation is gradually decreasing with increasing deposition, with the exception of the odd numbered peaks at 5 and 7 ML which are slightly higher than their even numbered neighbors. The difference between odd and even numbered peaks is, however, much smaller than in the case of growth at $300 \mathrm{~K}$. The decrease of the intensity of the peaks with increasing Fe deposition is due to an increasing step density arising from statistical fluctuations in the rate with which islands are formed on a lateral lengthscale determined by the mean diffusion length of the deposited Fe atoms. Such disorder can be annealed to a large extent by letting the film relax at or above the growth temperature (550-600 K). Figure 2 shows the time dependence of the specular intensity for a $5 \mathrm{ML}$ Fe film, which is annealed at $600 \mathrm{~K}$ directly after growing the film. For this film thickness and temperature, annealing of the disorder is completed in 5-10 min. The time necessary for annealing the disorder increases with film thickness and with decreasing anneal 


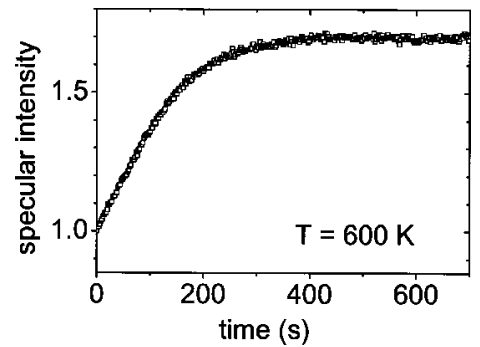

FIG. 2. The time dependence of the specular helium intensity of a $5 \mathrm{ML}$ Fe film grown at $550 \mathrm{~K}$ on $\mathrm{CoGa}(100)$ which is kept at a temperature of $600 \mathrm{~K}$.

temperature. However, by alternately depositing a few monolayers and annealing them, we have prepared Fe films with a thickness of more than $20 \mathrm{ML}$ on which oscillations are found during growth comparable to those at 7-8 ML in Fig. $1 .{ }^{16}$ There seems to be no reason which would prevent extension of this procedure to thicker films.

\section{B. Film stability}

As discussed above the quality of Fe films, with respect to surface order, improves with increasing growth and anneal temperature. However, the temperature range available for preparing such a film is limited because of intermixing. If the temperature becomes too high, $\mathrm{Fe}$ atoms may diffuse into the bulk of the CoGa crystal and $\mathrm{Ga}$ (or Co) atoms may diffuse into the Fe film. The thermal stability of the iron films was investigated by AES. A 10 ML Fe film was deposited on $\mathrm{CoGa}(100)$ at room temperature and it was subsequently heated. AES spectra were measured every $50 \mathrm{~K}$. Figure 3 shows the peak-to-peak intensities of the $\mathrm{Fe}(47 \mathrm{eV}), \mathrm{Co}(775$ $\mathrm{eV})$, and $\mathrm{Ga}(1070 \mathrm{eV})$ AES transitions. The Fe peak-to-peak intensity is normalized to the intensity measured on the 10 ML Fe film and the Co and Ga peak-to-peak intensities to the intensity measured on the clean substrate. The AES intensities remain constant until a temperature of $650 \mathrm{~K}$ is reached. Above this temperature the Co and $\mathrm{Ga}$ signals increase and the Fe signal decreases, though some of the iron signal re-

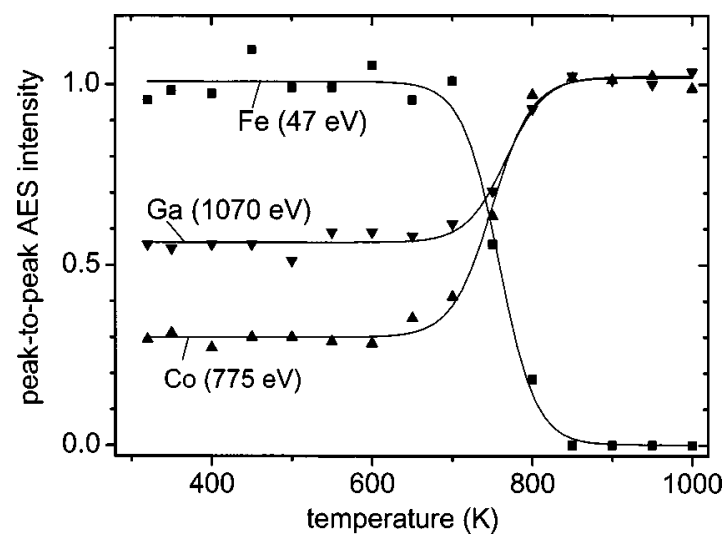

FIG. 3. Temperature dependence of the peak-to-peak AES intensities of the Auger transitions of $\mathrm{Fe}(47 \mathrm{eV}), \mathrm{Co}(775 \mathrm{eV})$, and $\mathrm{Ga}(1077 \mathrm{eV})$ of a $10 \mathrm{ML}$ Fe film deposited on $\mathrm{CoGa}(100)$ at $300 \mathrm{~K}$. The intensities are normalized.

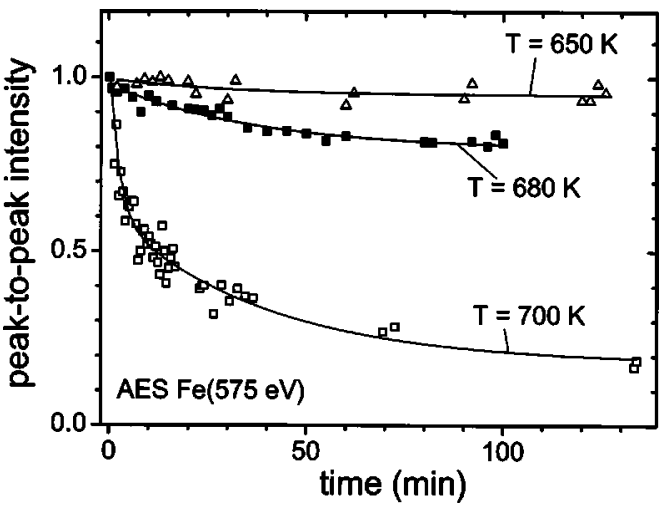

FIG. 4. Time dependence of the AES peak-to-peak intensity of the $\mathrm{Fe}(575 \mathrm{eV})$ transition of a $10 \mathrm{ML} \mathrm{Fe}$ film deposited at $550 \mathrm{~K}$ on $\mathrm{CoGa}(100)$, measured at temperatures of 650,680 , and $700 \mathrm{~K}$. The solid lines are drawn to guide the eye.

mains visible up to a temperature of $800 \mathrm{~K}$. Thus, intermixing of the Fe layer with the substrate starts around $650 \mathrm{~K}$. One may note the rather large $(>0.5)$ Ga peak-to-peak intensity measured on the Fe covered substrate. AES measurements on much thicker films still show a clear Ga AES transition, whereas the Co transition is absent in that situation. These results suggest that a Ga film [about 0.5-1 ML (Ref. 17)] is floating on the Fe film during growth.

In order to investigate the stability in more detail, isothermal relaxation experiments were performed at three temperatures between 650 and $700 \mathrm{~K}$. In Fig. 4 the peak-to-peak intensity of the high-energy (575 eV) AES transition is shown. At $650 \mathrm{~K}$ we do not observe a significant decrease of the intensity of the iron transition within two hours, so it is concluded that the Fe layer is essentially stable at this temperature. At $680 \mathrm{~K}$ a small but clear decrease of the AES Fe signal is found $(20 \%)$. Though the intermixing is far from complete, certainly on the considered time scale of $100 \mathrm{~min}$, the AES measurement does show that the film composition changes at this temperature. At $700 \mathrm{~K}$ only about $20 \%$ of the Fe layer remain visible by AES after $2 \mathrm{~h}$, i.e., the film composition has completely changed in this case. We conclude, therefore, that the iron film becomes unstable with respect to its composition at temperatures above $650 \mathrm{~K}$. To be on the safe side, we have limited the temperature in the other measurements reported in this work, on film growth and annealing (Sec. III A) and on the magnetic properties (Sec. III C), to the range $T<600 \mathrm{~K}$.

\section{Magnetic properties}

The magnetic properties of $0-10 \mathrm{ML}$ thick Fe films were investigated by polar and longitudinal Kerr effect measurements. All films considered were grown at $550 \mathrm{~K}$. Hysteresis loops were measured showing the Kerr ellipticity $\varepsilon$ as a function of the applied magnetic field, where $\varepsilon$ is proportional to the magnetization $M$. Figure 5 shows measurements of the Kerr ellipticity in the longitudinal configuration for Fe films with a coverage between 1 and $8 \mathrm{ML}$, with the magnetic field aligned along the [010] direction. The films were not annealed before measuring the hysteresis loops. Iron films 


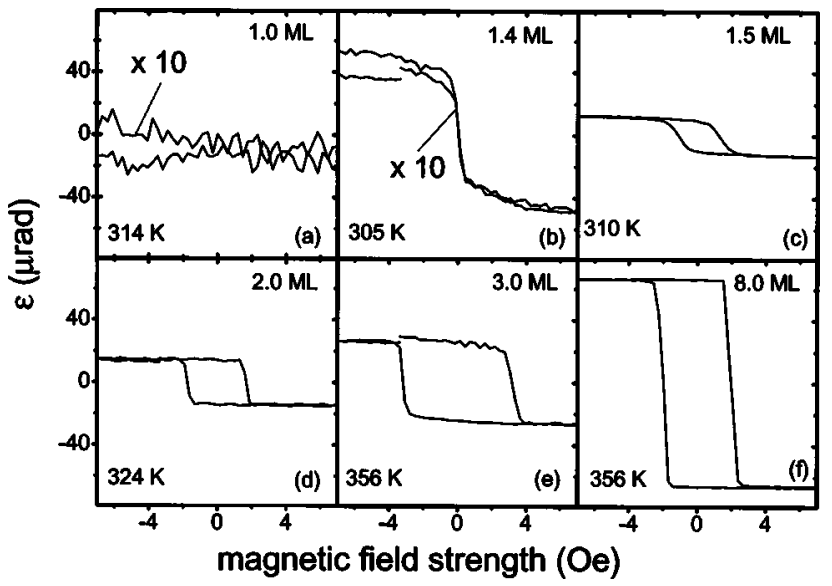

FIG. 5. Hysteresis loops measured by the magneto-optical Kerr effect in the longitudinal (in plane) configuration, on 1-8 ML thick Fe films deposited on CoGa(100) at $550 \mathrm{~K}$. (a) $1 \mathrm{ML}, T=314 \mathrm{~K}$, (b) $1.4 \mathrm{ML}, T=305 \mathrm{~K}$, (c) $1.5 \mathrm{ML}, T=310 \mathrm{~K}$, (d) $2.0 \mathrm{ML}, T$ $=324 \mathrm{~K}$, (e) $3.0 \mathrm{ML}, T=356 \mathrm{~K}$, (f) $8.0 \mathrm{ML}, T=356 \mathrm{~K}$. The scale of the ellipticity $\varepsilon$ in (a) and (b) is enlarged by a factor of 10 . The films grown at 3.0 and 8.0 ML were annealed at $600 \mathrm{~K}$ before the measurement.

grown at $550 \mathrm{~K}$ never showed a hysteresis of the Kerr ellipticity in the polar geometry up to a magnetic field strength of 600 Oe. Thus, the easy axis for magnetization of the films considered here is always parallel to the surface. (With other preparations conditions, a remanent magnetization perpendicular to the surface can be obtained ${ }^{17}$ ). From zero to $1 \mathrm{ML}$ Fe coverage we did not observe ferromagnetic behavior at $300 \mathrm{~K}$ as illustrated by the Kerr signal measured on a $1 \mathrm{ML}$ Fe film. At 1.4 ML the Kerr measurement shows a remanent ellipticity $\varepsilon_{r}>0$, i.e., there is a nonzero remanent magnetization $M_{r}$, but the coercive field $H_{c}$ is too small to be resolved around $T=300 \mathrm{~K}$. At $1.5 \mathrm{ML} \mathrm{Fe} \mathrm{coverage,} \mathrm{hysteresis} \mathrm{loops}$ are observed for which $\varepsilon_{r}$ and $H_{c}$ are both sufficiently large to be resolved. Upon further increasing the $\mathrm{Fe}$ coverage, the remanent magnetization $M_{r}$ increases and switching of the magnetization direction becomes more abrupt, as it is illustrated by the measurements on films with a thickness of 2, 3, and $8 \mathrm{ML}$. Based on the measured hysteresis loops, we estimate the Curie temperature to be $T_{c}=300 \mathrm{~K}$ for a Fe film with a coverage between 1.1 and 1.3 ML. An interesting phenomenon seen in the figure is that the coercive field $H_{c}$ increases with coverage between 1.4 and $3 \mathrm{ML}$, whereas at higher coverage $H_{c}$ decreases slightly as the film becomes thicker.

The temperature dependence of both $H_{c}$ and $M_{r}$ depends on the thickness as shown in Fig. 6 for films of 1.7 and 3 ML. The films were annealed at $550-600 \mathrm{~K}$ before the measurements. Figure 6(a) shows the temperature dependence of the coercive field $H_{c}$ and the remanent ellipticity $\varepsilon_{r}$ for a 1.7 ML Fe film. From the temperature dependence of $\varepsilon_{r}$ we can determine the Curie temperature of the $1.7 \mathrm{ML}$ film $T_{c}$ $=525 \mathrm{~K}$. The coercive field for the $1.7 \mathrm{ML}$ film is found to decrease about linearly with temperature up to $400 \mathrm{~K}$. Above $400 \mathrm{~K}$ the decrease of $H_{c}$ slows down, and at $460 \mathrm{~K}$, well below the Curie temperature, it has become so small that we

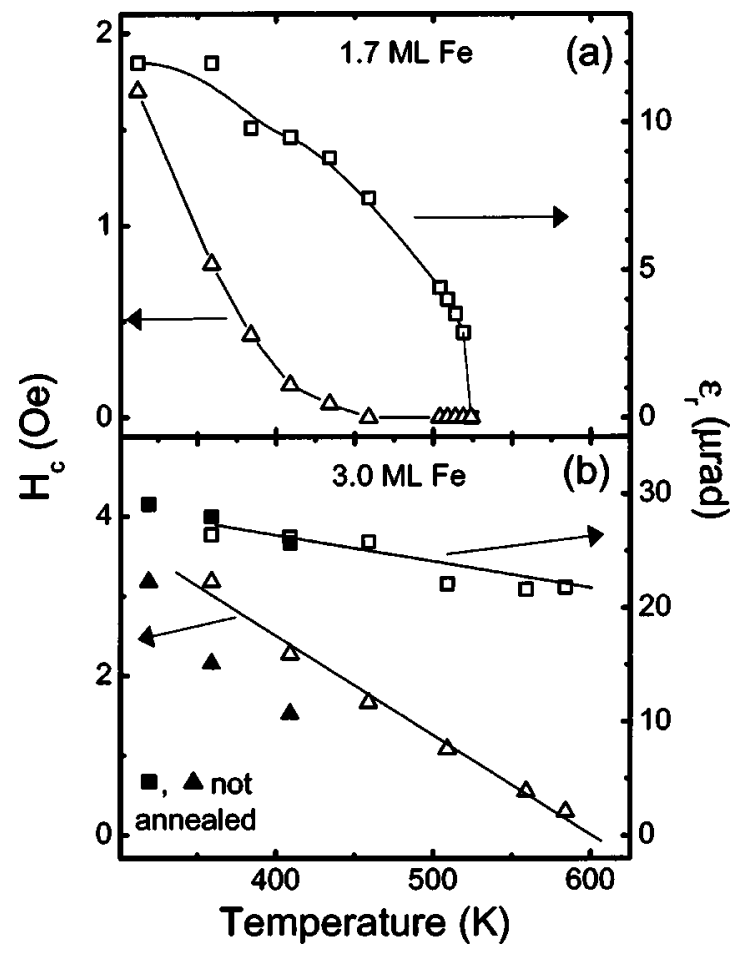

FIG. 6. Temperature dependence of the coercive field $H_{c}$ and the remanent ellipticity $\varepsilon_{r}$, corresponding to a remanent magnetization $M_{r}$, of Fe films deposited on $\mathrm{CoGa}(100)$ with a thickness of (a) $1.7 \mathrm{ML}$ and (b) $3 \mathrm{ML}$. The films were grown at $550 \mathrm{~K}$ and subsequently annealed at $600 \mathrm{~K}$ for $10-20 \mathrm{~min}$. For the $3 \mathrm{ML}$ film additional data are shown $(\boldsymbol{\square}, \boldsymbol{\Delta})$ which were measured on a film which was not annealed subsequent to growth.

cannot resolve it anymore. For the $3 \mathrm{ML}$ film [Fig. 6(b)] $T_{c}$ is larger than $600 \mathrm{~K}$ so we could not determine it without risking to change the film composition. For $H_{c}$ we observe again a linear decrease up to $600 \mathrm{~K}$, the vanishing of $H_{c}$ is, however, not seen because it occurs outside of the temperature range which is available to the experiment. It is interesting to note that the coercive field is found to depend on the ordering of the film. In Fig. 6(b) three measurements are shown for a film which was not annealed subsequent to growth. The value of $H_{c}$ obtained in these measurements ( $\left.\mathbf{\Delta}\right)$ lie clearly below the data measured for a fully relaxed film. In contrast, the ellipticity $\varepsilon_{r}$ measured on this film (ם) does not deviate significantly from the ellipticity measured on the annealed film. Thus, the remanent magnetization $M_{r}$ appears to be unaffected by the ordering of the film at $600 \mathrm{~K}$ subsequent to the growth.

\section{DISCUSSION}

We have grown very thin epitaxial bcc $\alpha$-Fe(100) films on $\mathrm{CoGa}(100)$, which are stable with respect to intermixing up to $650 \mathrm{~K}$ and which show in plane remanent magnetization at room temperature already at a thickness of $1.4 \mathrm{ML}$. In the considered temperature range $300 \mathrm{~K}<T<600 \mathrm{~K}$, layer by layer growth is observed. Evidence was found for a $0.5-1$ ML Ga layer floating on top of $\mathrm{Fe}$ films grown on $\mathrm{CoGa}(100)$. With respect to this, the growth of Fe films on 
$\mathrm{CoGa}(100)$ is similar to that on $\mathrm{Au}(100)$ and $\mathrm{Ag}(100)$ where also substrate atoms are found to float on the film surface. Fe films with a thickness of 3 ML or more, grown on $\mathrm{CoGa}(100)$ at $550 \mathrm{~K}$ and subsequently annealed at 550-600 $\mathrm{K}$, are very well ordered and smooth as evidenced by the very high intensity of the specular helium peak. During growth, oscillations of the specular helium intensity can be resolved up to a thickness of 20-25 ML. At $300 \mathrm{~K}$ the layer by layer growth is less ideal and can be resolved only up to $9 \mathrm{ML}$. At $300 \mathrm{~K}$ and a coverage larger than $2 \mathrm{ML}$ a clear difference is found between the growth of odd and even numbered layers, indicating that the bonding of $\mathrm{Fe}$ adatoms varies with the number of deposited layers. The observation of a smaller difference between growth of odd and even numbered layers at $550 \mathrm{~K}$ supports this interpretation.

Below a thickness of $3 \mathrm{ML}$ the TEAS experiments seem to indicate that the layer by layer growth is less ideal: the maximum expected at a deposition of $1 \mathrm{ML}$ is only showing up as a shoulder on the flank of the peak at 2 ML whereas this latter peak itself is observed but with a rather low intensity when compared to the maxima at 3-7 ML. The properties of the film, as probed by the helium beam, change dramatically in the thickness range of $0-3 \mathrm{ML}$. The reflectivity of a $1 \mathrm{ML} \mathrm{Fe}$ film is three times larger than that of the $\mathrm{CoGa}(100)$ substrate surface and upon increasing the $\mathrm{Fe}$ coverage subsequently to 3 ML the reflectivity is again increased by a factor of 3 for each added monolayer. It seems therefore unlikely that the deviating behavior observed below $3 \mathrm{ML}$ is only due to less ideal layer by layer growth than found at a coverage larger than $3 \mathrm{ML}$. The observed behavior could be due to changes in the bonding of $\mathrm{Fe}$ atoms in the first few deposited layers as discussed above in relation to growth between 3 and 9 ML. Both the reflectivity of the surface (structure factor) and the diffusion properties, which determine the growth characteristics, are expected to be sensitive to changes in the bonding of the $\mathrm{Fe}$ atoms.

Iron films, deposited on $\mathrm{CoGa}(100)$ at $550 \mathrm{~K}$, were found to have the easy axis for magnetization in the surface plane.

*Email address: 1.verheij@fz-juelich.de

${ }^{1}$ R. J. Hicken, S. J. Gray, A. Ercole, C. Daboo, D. J. Freeland, E. Gu, E. Ahmad, and J. A. C. Bland, Phys. Rev. B 55, 5898 (1997).

${ }^{2}$ Z. Q. Qiu, J. Pearson, and S. D. Bader, Phys. Rev. B 49, 8797 (1994).

${ }^{3}$ D. H. Bürgler, C. M. Schmidt, D. M. Schaller, F. Meisinger, R. Hofer, and H. J. Güntherodt, Phys. Rev. B 56, 4149 (1997).

${ }^{4}$ Y. Suzuki, T. Katayama, S. Yoshida, and K. Tanaka, Phys. Rev. Lett. 68, 3355 (1992).

${ }^{5}$ T. Kawagoe, T. Kotaki, T. Shibasaki, Y. Ohmori, and A. Itoh, Surf. Sci. 468, 1 (2000).

${ }^{6}$ O. S. Hernan, A. L. Vazquez de Parga, J. M. Gallego, and R. Miranda, Surf. Sci. 415, 106 (1998).

${ }^{7}$ S. D. Bader and E. R. Moog, J. Appl. Phys. 61, 3729 (1987).

${ }^{8}$ W. Dürr, M. Taborelli, O. Paul, R. Germar, W. Gudat, D. Pescia, and M. Landolt, Phys. Rev. Lett. 62, 206 (1989).

${ }^{9}$ Z. Q. Qiu, J. Pearson, and S. D. Bader, Phys. Rev. Lett. 70, 1006
At room temperature, the ferromagnetic behavior of the $\mathrm{Fe}$ films is found to start at a coverage between 1.1 to 1.3 ML. At a film thickness of 1.5 ML we are able to resolve a coercive field which is larger than zero. The Curie temperature is found to increase quite rapidly from $300 \mathrm{~K}$ at a coverage of 1.1-1.3 ML to $525 \mathrm{~K}$ for $1.7 \mathrm{ML}$ film. For a slightly thicker film (2 ML), $T_{c}$ has increased to above $600 \mathrm{~K}$ where it cannot be measured anymore without having the risk that the film composition is changed. For Fe films grown on $\mathrm{Au}(100)$, ferromagnetic behavior is also found at a coverage well below 2 ML. ${ }^{7,18}$ In principle, one would expect that at least two $\mathrm{Fe}$ monolayers are required for getting a ferromagnetic film, since in the Fe (100) plane only the next nearest neighbor sites are occupied. The reason for getting ferromagnetic behavior for Fe films on $\mathrm{CoGa}(100)$ well below a thickness of 2 ML could be due to a coupling between Fe and Co atoms at the film/substrate interface. Alternatively, nonideal layer by layer growth at a coverage below 2 ML could result in three-dimensional structures which are already large enough at a small coverage to show ferromagnetic behavior. At this stage we can not distinguish between the two possibilities since the TEAS measurements show that both bonding and the growth properties are different below a thickness of 3 ML. Also the observation of a coercive field which is increasing with coverage below 3 ML could be interpreted both ways since both a different bonding and an increased disorder could be the reason for a smaller value of $H_{c}$. Disorder of the film was found to result in a lower value of $H_{c}$ (see Fig. 6). The decrease of $H_{c}$ between 3 and 8 ML coverage can be explained therefore by an increasing disorder with film thickness, which is expected to be present due to a slower ordering process for thicker films. In contrast to $H_{c}$, the magnetization $M_{r}$ does not show a clearly different thickness dependence below and above a coverage of 3 ML.

\section{ACKNOWLEDGMENTS}

This work was supported by the HGF project Magnetoelectronics.
(1993).

${ }^{10}$ C. Liu and S. D. Bader, J. Vac. Sci. Technol. A 8, 2727 (1990).

${ }^{11}$ J. Shen, M. Drakaki, and J. L. Erskine, Phys. Rev. B 45, 3636 (1992).

${ }^{12}$ R. Franchy, M. Eumann, and G. Schmitz, Surf. Sci. 470, 337 (2001).

${ }^{13}$ F. M. Pan, C. Pflitsch, R. David, L. K. Verheij, and R. Franchy, Phys. Rev. B 63, 125414 (2001).

${ }^{14}$ F. M. Pan, L. K. Verheij, R. David, and R. Franchy, Thin Solid Films 400, 22 (2001).

${ }^{15}$ G. Schmitz, Ph.D. thesis, Heinrich Heine University Düsseldorf, Germany, 1999.

${ }^{16}$ C. Pflitsch, Ph.D. thesis, Heinrich Heine University Düsseldorf, Germany, 2002.

${ }^{17}$ T. Balster, D. A. Domokos, L. K. Verheij, and R. Franchy (unpublished).

${ }^{18}$ O. Paul, M. Taborelli, and M. Landolt, Surf. Sci. 211/212, 724 (1989). 